\title{
Patient-reported outcomes: opportunities and challenges in Central Europe
}

\author{
Márta Péntek ${ }^{1}$
}

Published online: 20 May 2019

○) Springer-Verlag GmbH Germany, part of Springer Nature 2019

Patient-reported outcomes (PRO) have become important instruments of healthcare and health policy making, as these can reflect patients' feedbacks on healthcare outcomes. PROs can be used to evaluate the performance of healthcare systems and to enhance benchmarking and quality improvement of healthcare services [1]. There are valuable systematic collections of health data on the local and EU level that are used to monitor health developments in most EU countries. However, classical descriptive health statistics need to be complemented by measures that can be interpreted by and incorporated in health economic evaluations of new technologies. Applying PROs can make a better use of these regularly assessed data to inform health policy decisions. PROs are also cornerstones of medical decision making. PROs play an increasing role in the drug registration process being primary or secondary endpoints of multicentric clinical trials. Initiation and cessation of treatments are based on PRO measurements in an increasing number of conditions, cutoff points are stated in international and national clinical and financial guidelines.

Despite the increasing need for PROs both on the national and European level, there is a scarcity of validated measurement tools and reference data in the Central and Eastern European (CEE) regions. Countries that have validated PRO measures and reference scores from relevant patient and population samples have better opportunities to participate in international strategic collaborations, health and innovation programmes, as well as in European health and healthcare research projects. The CEE region is lagging behind in this field compared to many other parts of Europe.

In this Supplement, we aim to report a series of recent PRO studies from Hungary, Poland and Slovenia and systematic reviews focusing on the CEE region.

Márta Péntek

marta.pentek@uni-corvinus.hu

1 Department of Health Economics, Corvinus University of Budapest, Fővám tér 8, Budapest 1093, Hungary
Ageing is a major challenge in Europe. Informal care provided by family members for individuals having self-care problems due to a disease or ageing is significant, especially in chronic conditions. Informal care can contribute substantially to the well-being of individuals with functional limitations but may influence caregivers' quality of life. The inclusion of informal care in cost-effectiveness evaluations can be decisive on the results [2]. Baji et al. assessed the quality of life of informal caregivers in Hungary, Poland and Slovenia. Care-related burden was measured by the preference-based CarerQol questionnaire alongside the EQ-5D-5L health status measure, providing comparable utility data for health economic analyses.

Mutual understanding between patients and healthcare providers, as well as patients' involvement in the formulation of therapeutic goals are key elements of effective and cost-effective healthcare. Clinicians and patients often have different views or preferences. Subjective over- or underestimation of future health by the patients can have significant impact on the evaluation of the therapeutic results measured by PROs and, as a consequence, on patients' actual health-related decisions [3]. Similarly, unrealistic subjective health expectations might retain people from considering their future needs for health- or long-term care and hamper their planning for older ages. Péntek et al. investigated individuals' subjective expectations regarding ageing, including provisions on health, happiness, labour force participation, future care needs and availability of care services. Standardised measurement tools were used to formulate questions on subjective expectations allowing comparisons with national statistics and population norms.

Previous studies in The Netherlands and Hungary have shown that people considered some health problems acceptable over a certain age, while the worst states were rejected at all ages $[4,5]$. The EQ-5D-3L questionnaire was used to learn the age-dependent acceptability of health problems. Zrubka et al. aimed to further develop this methodology and put it on a test in an empirical study in Hungary. 
In parallel with increasing abundancy and complexity of health information, the involvement of patients in healthrelated decisions has become an essential element of modern medicine. On the one hand, professional guidelines consider shared decision making (SDM) as one of the main principles of patient care. On the other hand, the Internet itself and digitalisation of healthcare have undoubtedly facilitated access to health information and made international sources directly available for the public. The possible misinterpretation of the information obtained without professional control can lead to under- or overuse of healthcare and self-medication, resulting in significant health loss and extra costs. Despite its significance, little is known about the population's approach to SDM, health information gathering habits and eHealth literacy in the CEE region and there is also a scarcity of standard measures in the international literature.

Rencz et al. developed and validated the Hungarian version of Shared Decision Making Questionnaire (SDM-Q9), Zrubka et al. implemented the Hungarian version of the eHealth Literacy Scale (eHEALS) and tested its psychometric properties in an online population-based survey in Hungary.

In the past years, besides the penetration of PRO measures as assessment tools in healthcare, patient-reported experience measures (PREMs) came into focus. PREMs are regularly reported by the OECD, however, there is a shortage of data on patients' experiences in the CEE region. Access to health care and patients' experiences in the outpatient sector have been studied in an online survey done in Hungary by Lucevic et al. and Brito Fernandes et al. using the standardised PREM tool recommended by the OECD. Results were compared to available data and the OECD average.

A set of valuable PRO studies were performed involving specific patient samples, focusing on costly diseases. There is an increasing body of literature arguing that the benefit of treatments expressed in quality adjusted life years (QALY) should be based on both patient and general public preferences in health technology assessments (HTA) [6]. In line with this concept, Rencz et al. compared patient and general population values for luminal and perianal fistulising Crohn's disease health states using the time-trade-off method. Recently, subjective well-being has gained increasing attention in health policy. Mitev et al. evaluated satisfaction with life in patients with pemphigus, and analysed its relationship with clinical severity and health-related quality of life (PRO) through a path analysis.

In most CEE countries, HTA has been implemented and QALY-based economic evaluations are required to support reimbursement decisions. The EQ-5D is by far the most commonly used preference-based measure to calculate QALYs in the region [7]. However, up-to-date population norm with the EQ-5D-3L is only available in Hungary, Poland and Slovenia. Moreover, country-specific value sets are available only in Poland and Slovenia in the CEE region (and studies are ongoing in another few). Therefore, generalizability of the data within the region is a main issue for countries with no country-specific norms, and not less important is a clear understanding of the impact of value set choice.

Zrubka et al. performed a detailed comparison of the three CEE EQ-5D-3L population norms and developed a common norm considering socio-demographic characteristics of the three countries. Furthermore, a comparative analysis of the 4 different EQ-5D-3L value sets (UK, Polish, Slovenian and European) was conducted for the assessment of 18 chronic illnesses. Weszl et al. pointed out in their systematic literature review that EQ-5D is the second most commonly applied PRO in device studies in Europe. However, few device studies including PROs were done in the Central and Eastern European regions. Prevolnik-Rupel et al. highlighted the limited use of the EQ-5D-5L version of the questionnaire in neurological diseases in the CEE region. This is in line with findings in other clinical fields and calls for new studies. The surveys detailed above involving the new EQ-5D-5L are valuable contributions to fill this gap.

A systematic literature review by Brodszky et al. involving eight CEE countries provides an insight into cost-ofillness studies in the region. Whilst productivity loss was quite often reported, informal care was included only in half of the studies indicating that the importance of this type of care is still underestimated. Heterogeneities across the studies hampered the comparability and transferability of the results, another important signal for the urgent need for standardisation and harmonisation of studies in the region.

In conclusion, despite the clear increase in interest in PROs both on the researchers' and the health policy makers' level in the CEE over the past years, there is still a long way to go. I personally strongly believe that if researchers from CEE countries are able and start to work systematically on PROs, in collaboration with each other and with key opinion leaders of the field forming a strategical network, these shortcomings can be transformed into qualities within the foreseeable future. The fact that a number of prestigious researchers from CEE countries and international experts have joined with this research and are writing this Supplement proves that this is the right way to go.

Acknowledgements This Supplement was supported by the Higher Education Institutional Excellence Program of the Ministry of Human Capacities in the framework of the 'Financial and Public Services' research project (20764-3/2018/FEKUTSTRAT) at Corvinus University of Budapest. 


\section{References}

1. Nelson, E.C., Eftimovska, E., Lind, C., Hager, A., Wasson, J.H., Lindblad, S.: Patient reported outcome measures in practice. BMJ 350, g7818 (2015). https://doi.org/10.1136/bmj.g7818

2. Krol, M., Papenburg, J., van Exel, J.: Does including informal care in economic evaluations matter? A systematic review of inclusion and impact of informal care in cost-effectiveness studies. Pharmacoeconomics 33(2), 123-135 (2015). https://doi.org/10.1007/ s40273-014-0218-y

3. Brouwer, W.B., van Exel, N.J.: Expectations regarding length and health related quality of life: some empirical findings. Soc Sci Med 61(5), 1083-1094 (2005). https://doi.org/10.1016/j.socsc imed.2005.01.008

4. Wouters, S., van Exel, N.J.A., Rohde, K.I.M., Vromen, J.J., Brouwer, W.B.F.: Acceptable health and priority weighting: Discussing a reference-level approach using sufficientarian reasoning. Soc Sci Med 181, 158-167 (2017). https://doi.org/10.1016/j.socsc imed.2017.03.051

5. Pentek, M., Rojkovich, B., Czirjak, L., Geher, P., Keszthelyi, P., Kovacs, A., Kovacs, L., Szabo, Z., Szekanecz, Z., Tamasi, L.,
Toth, A.E., Ujfalussy, I., Hever, N.V., Strbak, B., Baji, P., Brodszky, V., Gulacsi, L.: Acceptability of less than perfect health states in rheumatoid arthritis: the patients' perspective. Eur J Health Econ 15(Suppl 1), S73-82 (2014). https://doi.org/10.1007/ s10198-014-0596-2

6. Versteegh, M.M., Brouwer, W.B.F.: Patient and general public preferences for health states: a call to reconsider current guidelines. Soc Sci Med 165, 66-74 (2016). https://doi.org/10.1016/j. socscimed.2016.07.043

7. Rencz, F., Gulacsi, L., Drummond, M., Golicki, D., Prevolnik Rupel, V., Simon, J., Stolk, E.A., Brodszky, V., Baji, P., Zavada, J., Petrova, G., Rotar, A., Pentek, M.: EQ-5D in Central and Eastern Europe: 2000-2015. Qual Life Res 25(11), 2693-2710 (2016). https://doi.org/10.1007/s11136-016-1375-6

Publisher's Note Springer Nature remains neutral with regard to jurisdictional claims in published maps and institutional affiliations. 